\title{
Highlights of the Rome Workshop on gamma-ray bursts in the afterglow Era
}

\author{
D.Q. Lamb \\ Department of Astronomy and Astrophysics, University of Chicago, 5640 South Ellis Avenue, Chicago, IL 60637, U.S.A.
}

Received January 21; accepted March 22, 1999

\begin{abstract}
I review some of the highlights of the Rome Workshop on Gamma-Ray Bursts, and discuss some of the questions these results pose about the nature and origin of gamma-ray bursts.
\end{abstract}

Key words: gamma-rays: bursts

\section{Introduction}

As a result of the initiative and the effort of many people on the BeppoSAX team, we now live in the era of gamma-ray burst (GRB) afterglows. Having advocated for the High Energy Transient Explorer mission over the years, I know how unlikely it seemed to most astronomers that GRBs would produce detectable X-ray, let alone optical, afterglows. The mounting evidence from the Burst and Transient Source Experiment onboard the Compton Gamma-Ray Observatory that GRBs are extragalactic only seemed to strengthen this view. The subsequent discoveries that GRBs have X-ray, optical and radio afterglows has transformed the field. This workshop shows how great the impact is that these discoveries have had on the study of GRBs. The vast majority of the observational and theoretical results that were presented at this Workshop come from, or are motivated by, studies of the radio, optical and X-ray properties of afterglows and host galaxies, the latter identified by their positional coincidence with the afterglows.

Here I describe some of the highlights of the Workshop, and discuss some of the questions these results pose about the nature and origin of GRBs. Of necessity, this review reflects my personal point of view. Also, I cannot discuss all of the important observational and theoretical results reported at this meeting, given the limited space available. This summary is regretfully therefore incomplete.

\section{How many classes of GRBs are there?}

The discovery six months ago of an unusual Type Ic supernova, SN 1998bw (Galama et al. 1998; Galama 1999), in the BeppoSAX WFC error circle for GRB 980425 (Soffitta et al. 1998) (see Fig. 1) has focused attention once again on the question: How many distinct classes of GRBs are there?

If GRB 980425 were associated with SN 1998bw, the luminosity of the burst would be $\sim 10^{46} \mathrm{erg} \mathrm{s}^{-1}$ and its energy would be $\sim 10^{47} \mathrm{erg}$. These values are five orders of magnitude less than those of the other BeppoSAX bursts, whose luminosities range from $10^{50}$ to $10^{53} \mathrm{ergs} \mathrm{s}^{-1}$ and whose energies range from $10^{52}$ to $10^{55} \mathrm{ergs}$ (see below). Moreover, the behaviors of the X-ray and optical afterglows would be very different from those of the other BeppoSAX bursts, yet the burst itself is indistinguishable from other BeppoSAX and BATSE GRBs with respect to duration, time history, spectral shape, peak flux, and fluence (Galama et al. 1998).

There is another troubling aspect about the proposed association between GRB 980425 and SN 1998bw: Also inside the BeppoSAX WFC error circle was a fading X-ray source (Pian et al. 1998a,b; Pian et al. 1999; Piro et al. 1998) (see Fig. 1). Connecting this fading X-ray source with the burst gives a power-law index of $\sim 1.2$ for the temporal decay rate (Pian et al. 1998b), which is similar to the behavior of the other X-ray afterglows observed using BeppoSAX, ROSAT and ASCA. This fading X-ray source must therefore be viewed as a strong candidate for the X-ray afterglow of GRB 980425. There is also strong statistical evidence that all Type Ib-Ic supernovae (SNe) do not produce observable GRBs (Graziani et al. 1999a,b).

Approaching the possible association between SN 1998bw and GRBs from the opposite direction, one can ask: What fraction $f_{\text {GRB }}$ of the GRBs detected by BATSE could have been produced by Type Ib-Ic SNe, assuming that the proposed association between GRB 980425 and SN 1998bw is correct, and that the bursts produced are similar to GRB 980425? Assuming 


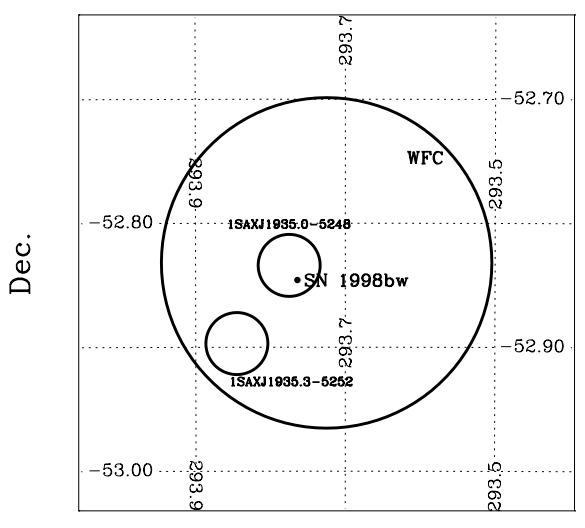

R.A.

Fig. 1. GRB 980425 field, showing the positional error circle for GRB 980425 determined using the BeppoSAX WFC (large solid circle), the positional error circles for the fading X-ray source detected by the BeppoSAX NFI (small solid circle labeled 1SAX1935.3-5252) and for the host galaxy of SN 1998bw (small solid circle labeled 1SAX1935.0-5248). From Graziani et al. (1999a)

that the association between SN1998bw and GRB 980425 is real, using this association to estimate the BATSE sampling distance for such events under the admittedly dubious assumption that the GRBs produced by Type Ib-Ic SNe are roughly standard candles, and assuming that all Type Ib-Ic SNe produce observable GRBs, Graziani et al. (1999a,b) find that no more than $\sim 90$ such events could have been detected by BATSE during the lifetime of the Compton Gamma-Ray Observatory, indicating that the fraction $f_{\mathrm{GRB}}$ of such events in the BATSE catalog can be no more than about $5 \%$. This result suggests that the observation of another burst like GRB 980425 is unlikely to happen any time soon, even assuming that the association is real, and consequently, the question of whether Type Ib-Ic SNe can produce extremely faint GRBs is likely to remain open for a long time.

Earlier studies have shown that gamma-ray bursts can be separated into two classes: short, harder, more variable bursts; and long, softer, smoother bursts (see, e.g., Lamb et al. 1993; Kouveliotou et al. 1993). Recently, Mukherjee et al. (1999) have provided evidence for the possible existence of a third class of bursts, based on these same properties of duration, hardness and smoothness properties of the bursts. Also, the hardest long bursts exhibit a pronounced deviation from the $-3 / 2$ power-law required for a homogeneous spatial distribution of sources, whereas the short bursts and the softest long bursts do not (Pizzichini 1995; Kouveliotou 1996; Belli 1997, 1999; Tavani 1998). These results contradict the expectation that the most distant bursts should be the most affected by cosmological energy redshift and time dilation. Some bursts show considerable high-energy $(E>300 \mathrm{keV})$ emission whereas others do not, but it is doubtful that this difference signifies two separate GRB classes, since a similar difference in behavior is seen for peaks within a burst (Pendleton et al. 1998).

It is not clear whether the short and long classes, and the other differences among various burst properties, reflect distinct burst mechanisms, or whether they are due to beaming-or some other property of the bursts-and different viewing angles. Some theorists say, however, that the "collapsar" or "hypernova" model cannot explain the short bursts (see, e.g., Woosley 1999).

Because of observational selection effects, all of the GRBs that have been detected by the BeppoSAX GRBM and observed by the WFC have been long bursts. It may be possible for BeppoSAX to revise its GRB detection algorithm in order to detect short bursts. We also expect that HETE-2 will detect short bursts and determine their positions (Kawai et al. 1999; Ricker et al. 1999). If so, follow-up observations may well lead to a breakthrough in our understanding of the nature of the short bursts similar to that which has occurred for the long bursts.

A nightmare I sometimes have is that HETE-2 provides accurate positions for a number of short bursts, but the positions are not coincident with any host galaxies because the bursts are due to merging compact object binaries that have drifted away from their galaxy of origin (see below). And furthermore, the bursts exhibit no soft X-ray, optical, or radio afterglows because any envelope that the progenitors of the compact objects might have expelled has been left behind, and the intergalactic medium is too tenuous to dissipate efficiently the energy in the relativistic external shock that is widely thought to be the origin of GRB afterglows. The redshifts of such bursts would be difficult, if not impossible, to determine, since they could not be inferred from the redshift of any host galaxy, nor constrained by the observation of absorption-line systems in the spectrum of any optical afterglow.

On a more positive note, future radio, optical, and X-ray observations of GRB afterglows and host galaxies, may well lead to the identification of new subclasses of GRBs.

\section{GRB host galaxies}

The detection of burst X-ray and optical afterglows has led in eight cases to identification of the likely host galaxy by positional coincidence with the optical afterglow. At $R=25.5-26$, the typical $R$-band magnitudes of these galaxies, galaxies cover $10-15 \%$ of the sky for groundbased observations, because of smearing of the galaxy images due to seeing. Therefore one expects $1 / 10$ to $1 / 7$ of ground-based "identifications" to be incorrect. If we are lucky, all of the identifications made to date are correct, but if we are unlucky, one or two are incorrect. On the other hand, it is highly probable that all of the host 


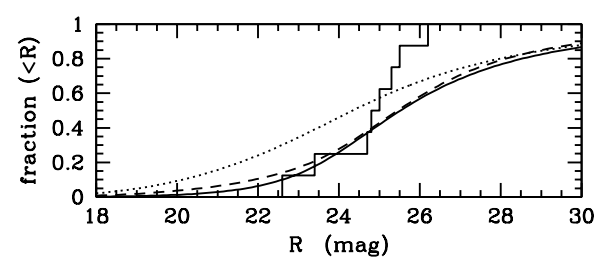

Fig. 2. Cumulative distribution of extinction-corrected $R$-band magnitudes of the eight GRB host galaxies identified so far (solid histogram), compared to that expected for the fiducial star-formation-rate model (solid curve), total-stellar-density model (dotted curve) and constant-comoving-volume model (dashed curve). From Hogg \& Fruchter (1999)

galaxies identified from HST observations are correct (e.g., the host galaxies for GRBs 970228, 970508, 971214, and 980329), since HST images are free of the effects of seeing that bedevil observations from the ground. It is also reassuring that in two cases (GRBs 970508 and 971214), the host galaxy identified from ground-based observations has been confirmed by later HST observations.

Let me mention a related concern. Until very recently, all GRB host galaxies had $R=25.7 \pm 0.3$, no matter what their redshift and no matter how the afterglow on which the identification is based was discovered (i.e., whether detected in the optical, NIR, or radio); that is, the $R$ band magnitude of the GRB host galaxy appeared to be a kind of "cosmological constant". In contrast, if the GRB rate is proportional to the star formation rate (SFR) (see below), one expects a relatively broad distribution of $R$-band magnitudes for GRB host galaxies (Hogg \& Fruchter 1999; Fruchter 1999; Madau 1999) (see Fig. 2). The recent discovery of the host galaxy of GRB 980703 at $R=22.6$ broadens the observed distribution of host galaxy $R$-band magnitudes, provided the identification is correct. However, it also increases the asymmetry of the $R$-band magnitude distribution, which exhibits a tail toward the bright end and a cutoff toward the dim. This is the opposite of what one expects if the GRB rate is proportional to the SFR.

This raises the possibility that in some cases we are merely finding the first galaxy along the line-of-sight to the burst. If so, in some cases the galaxy found may be a foreground galaxy, and the actual host galaxy may lie behind it. Or it might be that the GRB rate is not proportional to the SFR (see the discussion below). Or most likely of all, the asymmetry may merely reflect the fact that we are still very much in the regime of small number statistics. Additional confirmations and/or identifications of host galaxies using HST will resolve this question.

Castander \& Lamb (1998) showed that the light from the host galaxy of GRB 970228, the first burst for which $\mathrm{X}$-ray and optical afterglows were detected, is very blue, implying that the host galaxy is undergoing copious star formation and suggesting an association between GRB sources and star forming galaxies. Subsequent analyses of

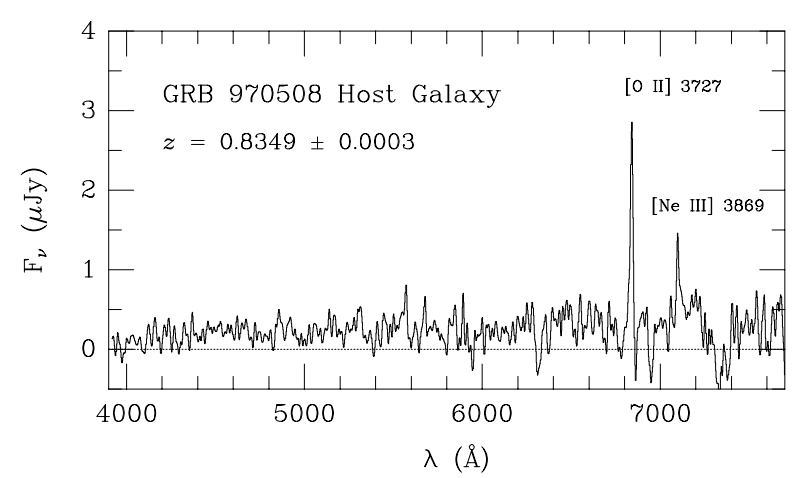

Fig. 3. The weighted average spectrum of the host galaxy of GRB 970508. Prominent emission lines of [O II] and [Ne III] are labeled. From Bloom et al. (1998)

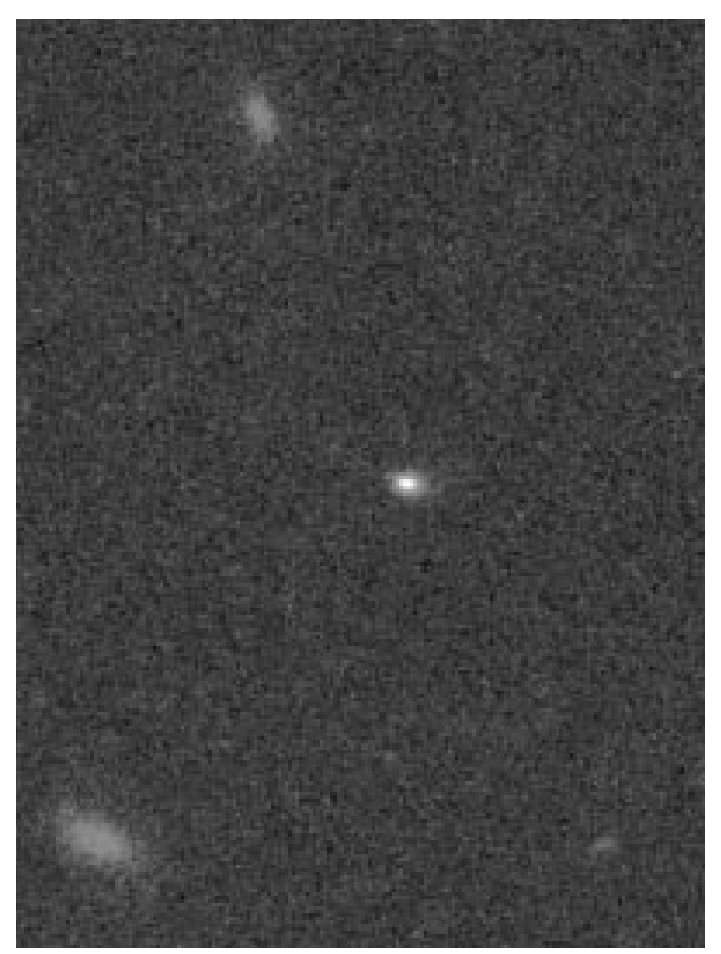

Fig. 4. HST image of the afterglow and host galaxy of GRB 970508 taken 1998 August. From Fruchter (1999)

the color of this galaxy (Castander \& Lamb 1999; Fruchter et al. 1999; Lamb et al. 1999) and other host galaxies (see, e.g., Kulkarni et al. 1998; Fruchter 1999) have strengthened this conclusion, as does the morphology and the detection of $[\mathrm{OII}]$ and Ly $\alpha$ emission lines from several host galaxies (see, e.g., Metzger et al. 1997b; Kulkarni et al. 1998; Bloom et al. 1998) (see Fig. 3). The positional coincidences between several burst afterglows and the bright blue regions of the host galaxies (see Fig. 4), and the evidence for extinction by dust of some burst afterglows (see, e.g., Reichart 1998; Kulkarni et al. 1998; Lamb et al. 1999), suggests that these GRB sources lie near or in the starforming regions themselves. 
The inferred size $(R \lesssim 1-3 \mathrm{kpc})$ and the morphology of GRB host galaxies strongly suggest that they are primarily low-mass $\left(M \lesssim 0.01 M_{\text {Galaxy }}\right)$ but not necessarily sub-luminous galaxies, because of the ongoing star formation in them (most have $L \lesssim 0.01-0.1 L_{\text {Galaxy }}$, but some have $L \sim L_{\text {Galaxy }}$; here $M_{\text {Galaxy }}$ and $L_{\text {Galaxy }}$ are the mass and luminosity of a galaxy like the Milky Way). A point sometimes not fully appreciated is that, while the total star formation rate in GRB host galaxies is often modest (resulting in modest [OII] and Ly $\alpha$ emission line strengths), the star formation per unit mass in them is very large.

\section{GRB distance scale and rate}

The breakthrough made possible by the discovery that GRBs have X-ray (Costa et al. 1997), optical (Galama et al. 1997) and radio (Frail et al. 1997) afterglows cannot be overstated. The discovery by Metzger et al. (1997a) of redshifted absorption lines at $z=0.83$ in the optical spectrum of the GRB 970508 afterglow showed that most, perhaps all, GRB sources lie at cosmological distances. Yet we must remember that GRB 970508 remains the only GRB whose distance we have measured directly. The current situation is summarized below, in order of increasing uncertainty in the redshift determination.

In the cases of two other bursts, GRB 980703 (Bloom et al. 1999) and GRB 971214 (Kulkarni et al. 1998; Kulkarni 1999), we infer the redshifts $(z=0.96$ and 3.42) of the bursts from the redshift of a galaxy coincident with the burst afterglow (and therefore likely to be the host galaxy - but recall my earlier comments).

In the case of a fourth burst, GRB 980329, a redshift $z \approx 5$ was inferred by attributing the precipitous drop in the flux of the optical afterglow between the $I$ - and $R$-bands to the Lya forest (Fruchter 1999; Lamb et al. 1999). However, Djorgovski et al. (1999) recently reported that this burst must lie at a redshift $z<3.9$, based on the absence of any break longward of $6000 \AA$ in the spectrum of the host galaxy.

In the case of GRB 980703 (Piro 1999) and of a fifth burst, GRB 980828 (Yoshida 1999), there are hints of an emission-like feature in the X-ray spectrum, which, if interpreted as a redshifted Fe K-shell emission line, would provide redshift distances for these bursts. However, substantial caution is in order because the statistical significance of these features is slight. Indeed, all three "indirect" means of establishing the redshift distances of GRBs need verification by cross-checking the redshift distances found using these methods against those measured directly using redshifted absorption lines in the optical spectra of their afterglows. One arcminute or better angular positions in near real-time, like those that HETE-2 will provide (Ricker et al. 1999; Kawai et al. 1999), will greatly facilitate this task.
The table below summarizes the current situation, in order of increasing uncertainty in the redshift determination:

Redshifts of Afterglows 1

Redshifts of Coincident Galaxies 2

Redshifts from Afterglow Broad-Band Spectra 0

(?)

Redshifts from Fe Lines in X-Ray Afterglows (??) 0

Total

Even with the paucity of GRB redshift distances currently known, and the uncertainties about these distances, it is striking how our estimate of the GRB distance scale continues to increase. Not so long ago, adherents of the cosmological hypothesis for GRBs favored a redshift range $0.1 \lesssim z \lesssim 1$, derived primarily from the brightness distribution of the bursts under the assumption that GRBs are standard candles. (Of course, adherents of the galactic hypothesis argued for much smaller redshifts!). Now we routinely talk about redshift distances in the range $2 \lesssim z \lesssim 6$, and such a redshift range is supported by the three burst redshifts that have been determined so far.

Much of the motivation for considering such a redshift range for GRBs comes from the appealing hypothesis that the GRB rate is proportional to the star-formation rate (SFR) in the universe, an hypothesis that arose partly in response to the accumulating evidence, described earlier, that GRBs occur in star-forming galaxies, and possibly near or in the star-forming regions themselves. How far have we been able to go in testing this hypothesis? The answer: Not very far. First of all, as Madau (1999) discussed at this meeting, our knowledge of the SFR as a function of redshift is itself as yet poorly known. The few points derived from the relatively small Hubble Deep Field may not be characteristic of the SFR in the universe at large, not to mention concerns about star-forming galaxies at high redshift whose light might be extinguished by dust in the star-forming galaxies themselves, as well as uncertainties in the epoch and magnitude of star formation in elliptical galaxies. Second, we have redshift determinations for only three GRBs and $R$-band magnitudes for only eight GRBs. Much further work establishing the star formation rate as a function of redshift in the universe, as well as the redshift distances for many more GRBs, will be needed before this hypothesis can really be tested.

One thing is now clear: GRBs are a powerful probe of the high- $z$ universe. GRB 971214 would still be detected by BATSE and would be detected by HETE- 2 at a redshift distance $z \approx 10$, and it would be detected by Swift (whose sensitivity threshold is a factor of 5 below that of BATSE and HETE-2) at $z \approx 20$ ! If GRBs are produced by the collapse of massive stars in binaries, one expects them to occur out to redshifts of at least $z \approx 10-12$, the redshifts at which the first massive stars are thought to have formed, which are far larger than the redshifts expected for the most distant quasars. The occurrence of 
GRBs at these redshifts may give us our first information about the earliest generation of stars; the distribution of absorption-line systems in the spectra of their infrared afterglow spectra will give us information about both the growth of metallicity at early epochs and the large-scale structure of the universe, and the presence or absence of the Lyman- $\alpha$ forest in the infrared afterglow spectra will place constraints on the Gunn-Peterson effect and may give us information about the epoch at which the universe was re-ionized (Lamb \& Reichart 1999a).

The increase in the GRB distance scale also implies that the GRB phenomenon is much rarer than was previously thought. This implication has been noted at this meeting by Schmidt (1999), who finds that the GRB rate must be

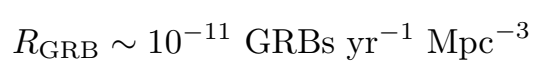

in order both to match the brightness distribution of the bursts and to accommodate the redshift distance of $z=3.42$ inferred for GRB 971214 .

By comparison, the rates of neutron star-neutron star (NS - NS) binary mergers (Totani 1999) and the rate of Type Ib-Ic supernovae (Cappellaro et al. 1997) are

$R_{\mathrm{NS}-\mathrm{NS}} \sim 10^{-6}$ mergers $\mathrm{yr}^{-1} \mathrm{Mpc}^{-3}$

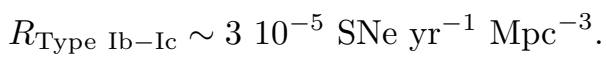

The rate of neutron star-black hole ( $\mathrm{NS}-\mathrm{BH}$ ) binary mergers will be smaller. Nevertheless, it is clear that, if either of these events are the sources of GRBs, only a tiny fraction of them produce an observable GRB. Even if one posits strong beaming (i.e., $f_{\text {beam }} \approx 10^{-2}$; see below), the fraction is small:

$$
\begin{aligned}
& R_{\mathrm{GRB}} / R_{\mathrm{NS}-\mathrm{NS}} \sim 10^{-3}\left(f_{\text {beam }} / 10^{-2}\right)^{-1} \\
& R_{\mathrm{GRB}} / R_{\text {Type Ib } / \mathrm{c}} \sim 310^{-5}\left(f_{\text {beam }} / 10^{-2}\right)^{-1} .
\end{aligned}
$$

Therefore, if such events are the sources of GRBs, either beaming must be incredibly strong $\left(f_{\text {beam }} \sim 10^{-5}-10^{-3}\right)$ or only rarely are the physical conditions necessary to produce a GRB satisfied. Can any theoretical astrophysicist be expected to explain such incredible beaming, or alternatively, such a non-robust, "flaky" phenomenon? I have a solution - at least in the case of SNe: We theorists merely need define those supernovae that produce GRBs to be a new class of SNe (Type $\mathrm{I}_{\text {grb }} \mathrm{SNe}$ ), and then challenge the observers to go out and find the other observational criteria that define this class!

\section{Implied energies and luminosities}

The maximum energy $\left(E_{\mathrm{GRB}}\right)_{\max }$ that has been observed for a GRB imposes an important requirement on GRB models, and is therefore of great interest to theorists. $\left(E_{\mathrm{GRB}}\right)_{\max }$ has increased as the number of GRB redshift distances that have been determined has increased. Currently, the record holder is GRB 971214 at $z=3.4$, which implies $E_{\mathrm{GRB}} \sim 510^{53}$ erg from its gamma-ray fluence, assuming isotropic emission and $\Omega_{\mathrm{M}}=0.3$ and $\Omega_{\Lambda}=0.7$ (Kulkarni 1999).

The table below summarizes the redshifts and energies of the bursts for which these are currently known:

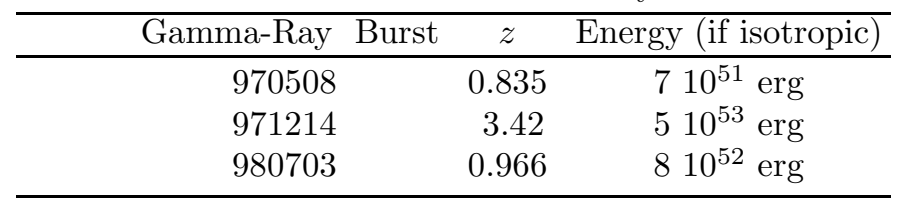

This kind of energy is difficult to accommodate in NS - NS or NS - BH binary merger models without invoking strong beaming. "Collapsar" or "hypernova" models have an easier time of it, and can perhaps reach $\sim 10^{54} \mathrm{erg}$ without invoking strong beaming by assuming a high efficiency for the conversion of gravitational binding energy into gamma-rays (Woosley 1999).

Both classes of models can be "saved" by invoking strong beaming $\left(f_{\text {beam }} \sim 1 / 10-1 / 100\right.$ (but see the lack of evidence of beaming discussed below). Even if GRBs are strongly beamed, they are still far and away the brightest electromagnetic phenomenon in the Universe, as the following comparison illustrates:

$$
\begin{aligned}
& \text { - } L_{\mathrm{SNe}} \lesssim 10^{44} \mathrm{erg} \mathrm{s}^{-1} \\
& \text { - } L_{\mathrm{SGR}} \lesssim 10^{45} \mathrm{erg} \mathrm{s}^{-1} \\
& \text { - } L_{\mathrm{AGN}} \lesssim 10^{45} \mathrm{erg} \mathrm{s}^{-1} \\
& \text { - } L_{\mathrm{GRB}} \sim 10^{51}\left(f_{\text {beam }} / 10^{-2}\right) \mathrm{erg} \mathrm{s}^{-1} \text {. }
\end{aligned}
$$

The luminosities of GRB 970508 and GRB 971214 differ by a factor of about one hundred. Thus (if there was previously any doubt), determination of the redshift distances for just three GRBs has put to rest once and for all the idea that GRBs are "standard candles." The extensive studies by Loredo \& Wasserman (1998a,b) and the study by Schmidt (1999) reported at this workshop show that the luminosity function for GRBs can be, and almost certainly is, exceedingly broad, with $\Delta L_{\mathrm{GRB}} / L_{\mathrm{GRB}} \gtrsim 10^{3}$. The results of Loredo \& Wasserman (1998a,b) show that the burst luminosity function could be far broader; and indeed, if GRB 980425 is associated with SN 1998bw, $\Delta L_{\mathrm{GRB}} / L_{\mathrm{GRB}} \gtrsim 10^{5}$.

Even taking a luminosity range $\Delta L_{\mathrm{GRB}} / L_{\mathrm{GRB}} \gtrsim 10^{3}$ implies that $\Delta F_{\mathrm{GRB}} / F_{\mathrm{GRB}} \gtrsim 10^{4}$, given the range in the distances of the three GRBs whose redshifts are known. This is far broader than the range of peak fluxes in the BASTE GRB sample, and implies that the flux distribution of the bursts extends well below the BATSE threshold.

The enormous breadth of the luminosity function of GRBs suggests that the differences (such as time stretching and spectral softening) between the apparently bright and the apparently dim bursts are due to intrinsic differences between intrinsically bright and faint bursts, rather than to cosmology.

Finally, a broad luminosity function is naturally expected in models with ultra-relativistic radial outflow and 
strong beaming (jet-like behavior). But then why is no large special relativistic Doppler redshift seen in GRB spectra; i.e., why is the spread in $E_{\text {peak }}$ so narrow?

\section{Burst models}

NS - NS or NS - BH binary mergers and "collapsar" or "hypernova" events continue to be the leading models for the energy source of GRBs. Rees (1999) described what he termed the "best buy" model, which involves a NS - BH binary merger and a magnetically powered jet. Woosley (1999) reported a series of calculations and hydrodynamic simulations that explore various stages of the collapsar scenario, including the production of a hydrodynamic jet (although the jet might also be magnetically powered in this scenario, if magnetic fields were included).

The increasingly strong evidence that the bursts detected by BeppoSAX originate in galaxies undergoing star formation, and may occur near or in the star-forming regions themselves, favors the collapsar model and disfavors the binary merger model as the explanation for long, softer, smoother bursts. Simulations of the kicks given to $\mathrm{NS}-\mathrm{NS}$ and NS - BH binaries by the SNe that form them shows that most binary mergers are expected to occur well outside any galaxy (Bulik \& Belczynski 1999). This is particularly the case, given that the GRB host galaxies identified so far have small masses, as discussed earlier, and therefore low escape velocities. The fact that all of the optical afterglows of the BeppoSAX bursts are coincident with the disk of the host galaxy therefore also disfavors the binary merger model as the explanation for the long, softer, smoother bursts.

Current models of the bursts themselves fall into three general categories: Those that invoke a central engine, those that invoke internal shock waves in a relativistically expanding wind, and those that invoke a relativistic external shock wave. Dermer (1999) argued that the external shock wave model explains many of the observed properties of the bursts. By contrast, Fenimore (1999; see also Fenimore et al. 1999) argued that several features of GRBs, such as the large gaps seen in burst time histories, cannot be explained by the external shock wave model, and that the bursts must therefore be due either to a central engine or to internal shocks in a relativistically expanding wind. Either way, the intensity and spectral variations seen during the burst must originate at a central engine. This implies that the lifetime of the central engine must in many cases be $t_{\text {engine }} \gtrsim 100-1000 \mathrm{~s}$, which poses a severe difficulty for $\mathrm{NS}-\mathrm{NS}$ or NS - BH binary merger models, if such models are invoked to explain the long, softer, smoother bursts, and may pose a problem for the collapsar model. Fenimore (1999) reported at this meeting that he finds no evidence of relativistic expansion in the time histories and spectra of the GRBs themselves, presenting a possible difficulty for the internal shock wave model.

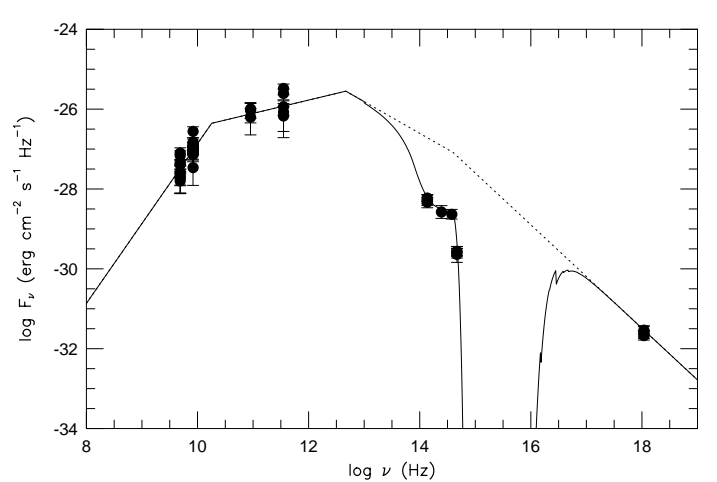

Fig. 5. The radio through X-ray spectrum of the afterglow of GRB 980329. All measurements have been scaled to a common time, approximately three days after the GRB. The solid curve is the best fit spectrum for an isotropic fireball that expands into a homogeneous external medium, extincted by dust at a redshift of $z=3.5$. The dotted curve is the un-extincted spectrum. From Lamb et al. (1999)

One puzzle about the bursts themselves is: Why are GRB spectra so smooth? The shock synchrotron model agrees well with observed burst spectra. But this agreement is surprising, since strong deviations from the simplest spectral shape are expected due to inverse Compton scattering, and if forward and reverse shock contributions to the prompt gamma-ray emission occur simultaneously or at different times (Tavani 1999).

Another puzzle is: Why is the spread in the peak energy $E_{\text {peak }}$ of the burst spectra so narrow? In the external shock model, this requires that all GRBs have nearly the same ultra-relativistic value of $\Gamma$. The narrow range in $E_{\text {peak }}$ is, if anything, more difficult to understand in the internal shock model. If $\Delta \Gamma / \Gamma \ll 1$ in the relativistic outflow, the range in $E_{\text {peak }}$ will be narrow, but then it is hard to understand why most of the energy of the relativistic outflow is dissipated during the burst rather than in the afterglow. Conversely, if $\Delta \Gamma / \Gamma \gg 1$ in the relativistic outflow, most of the energy of the relativistic outflow is dissipated during the burst rather than in the afterglow, but then one expects a wide range of $E_{\text {peak }}$ 's. This is a hint - like the problem discussed earlier that one would expect strong beaming to produce a large special relativistic Doppler redshift, yet this is not seen in burst spectra - that there may be something missing in our picture of the dissipation and radiation mechanisms in GRBs.

\section{Beaming}

Most theorists expect GRBs to be significantly beamed - many energetic astrophysical phenomena are (examples include young protostars; the so-called "microquasars", which are black hole binaries in the Galaxy; radio galaxies; and AGN). And theorists desire beaming because it saves their models. Several speakers at this workshop have emphasized these points (see, e.g., Dar 1999; Fargion 1999, 
and Rees 1999). Strong beaming probably requires strong magnetic fields, but no detailed physical model of how this might happen has been put forward as yet.

One can ask: Where is the observational evidence for beaming...? Fenimore (1999) told us that there is none in the time histories of the bursts themselves. Worse yet, Greiner (1999) reported that $f_{\mathrm{GRB}} / f_{\text {afterglow }}^{\mathrm{X}-\text { ray }} \gtrsim 1$ from an analysis of the ROSAT all sky survey. This constraint may not be as strong as it appears, because the duration of the temporal exposure in the ROSAT all-sky survey is only a few hundred seconds, and thus the sensitivity of the survey is relatively poor. Consequently, soft X-ray afterglows would be detectable by the ROSAT all-sky survey only within a day or so after the burst, when (in the relativistic external shock model of afterglows - see below) the soft X-ray emission is still highly beamed.

Constraints on so-called "orphan" optical afterglows, and therefore on the beaming of GRBs, will be strengthened by new low- $z$ SN Ia searches that will soon be underway. These searches will monitor an area of the sky that is roughly ten times larger than that monitored by current high- $z$ SN Ia searches down to the same limiting magnitude $\left(m_{B} \approx 20\right)$ (Perlmutter 1999).

\section{Afterglow models}

The simple fireball model (i.e., a spherically symmetric relativistic external shock wave expanding into a homogeneous medium) has been "in and out of the hospital" for months, but notices of its death appear to be premature. This amazes me, given the wealth of complexities one can easily imagine in the fireball itself and in its environment (Mészáros 1999). If the simple relativistic fireball model (or even more complex variants of it) suffice to explain burst afterglows (see Fig. 5), much can be learned, including the energy of the fireball per unit solid angle, the energy in the magnetic field, the energy in the relativistic electrons, and the density of the external medium into which the fireball expands (Wijers \& Galama 1999; van Paradijs 1999; Lamb et al. 1999). It should be possible, in principle, to use the effects on the afterglow spectrum of extinction due to dust in the host galaxy and of absorption by the Lyman- $\alpha$ forest to determine the redshift of the burst itself, but so far, this goal has eluded modelers (see, e.g., Lamb et al. 1999).

Currently, we are in the linear regime in terms of what we learn from each individual afterglow because, given the diversity of GRBs, GRB afterglows, and host galaxies, we have yet to sample the full "phase space" of afterglow or host galaxy properties. Still less have we sampled the full "phase space" of combinations of burst, afterglow, and host properties.

At the same time, we are in the strongly non-linear regime, in terms of what we learn from each individual observation of a burst afterglow. The value of each astronomer's observation is enhanced by the observations made by all other astronomers. As we have heard from several speakers at this workshop, the amount of information that can be gleaned from a given afterglow depends greatly on the number of measurements that exist both simultaneously in time and in wavelength, from the radio through the millimeter, sub-millimeter, near-infrared, optical, and $\mathrm{X}$-ray bands. Furthermore, since the range of redshifts for the bursts (and therefore also their afterglows) is large, we cannot know in advance which bands will be crucial. Thus simultaneous or near-simultaneous multi-wavelength observations of burst afterglows are essential, and therefore observations by as many observers as possible must be encouraged. Finally, greater co-operation and co-ordination among observers is important, and should be facilitated, as has been done by setting up the invaluable service represented by the Gamma-Ray Burst Coordinate Network (GCN) (Barthelmy et al. 1999).

\section{Star-forming regions}

Star forming regions consist of a cluster of O/B stars that lie in and around a clumpy cloud of dust and gas. We expect $A_{V} \gg 1$ for $\mathrm{O} / \mathrm{B}$ stars embedded in the cloud, and $A_{V} \approx 0$ for $\mathrm{O} / \mathrm{B}$ stars that have drifted out of the cloud and/or lie near the surface of the cloud and have expelled the gas and dust in their vicinity. Thus the optical/UV spectrum of star forming regions is a sum of the spectra of many hot (blue) stars, some of which are embedded in the cloud, and therefore heavily extinguished, and some of which lie on the surface or around the cloud, and are therefore essentially un-extinguished. This composite spectrum is rather blue, and yields a value $A_{V}^{\text {eff }} \approx 1$ when a single extinction curve is fitted to it.

The situation is very different when we consider an individual line-of-sight, as is appropriate for the afterglow of a GRB. If the GRB source lies outside and far away from any star-forming region, we expect $A_{V}^{\text {afterglow }} \lesssim 1$; if the GRB source lies outside but near a star-forming region, we expect $A_{V}^{\text {afterglow }} \lesssim 1$ about half the time and $A_{V}^{\text {afterglow }} \gg 1$ about half the time. Finally, if the GRB source is embedded in the star-forming region, we expect $A_{V}^{\text {afterglow }} \gg 1$.

Thus, if GRB sources actually lie in star-forming regions, one would expect $A_{V}^{\text {afterglow }} \gg 1$ (values of $A_{V}$ $\sim 10-30$ are not uncommon for dense, cool molecular clouds in the Galaxy). Is this consistent with what we see? No. However, this may not mean that GRB sources do not lie in star-forming regions. The reason is that the soft X-rays and the UV radiation from the GRB and its afterglow are capable, during the burst and immediately afterward, of vaporizing all of the dust in their path (Lamb \& Reichart 1999b). Thus the value of $A_{V}^{\text {afterglow }}$ that we measure may have nothing to do with the pre-existing value of the extinction through the star-forming region in which the burst source is embedded, but may instead 
reflect merely the extinction due to dust and gas in the disk of the host galaxy.

The GRB, and its soft X-ray and UV afterglow, are also capable of ionizing gas in any envelope material expelled by the progenitor of the burst source and in the interstellar medium of the host galaxy. This will produce Strömgren spheres or very narrow cones (if the burst and its afterglow are beamed) in hydrogen, helium and various metals (Bisnovatyi-Kogan \& Timokhin 1998; Timokhin \& Bisnovatyi-Kogan 1999; Mészáros 1999). Recombination of the ionized hydrogen eventually produces intense [CII], $[\mathrm{CIV}],[\mathrm{OVI}]$ and $[\mathrm{CIII}]$ emission lines in the UV, and intense $\mathrm{H} \alpha$ and $\mathrm{H} \beta$ emission lines in the optical. However, the line fluxes may still not be strong enough to be detectable at the large redshift distances of GRB host galaxies. Interaction of the GRB and its soft X-ray afterglow with any envelope material expelled by the progenitor of the burst source and with the surrounding interstellar medium can also produce intense fluorescent iron line emission (see, e.g., Mészáros 1999), but it is again difficult to see how the line flux could be large enough to be detectable or to explain the hints of a fluorescent iron emission line in the X-ray afterglows of GRB 980703 (Piro et al. 1999) and GRB 980828 (Yoshida et al. 1999).

\section{Future}

Each person has their own favorite list of future observational needs. Here is mine:

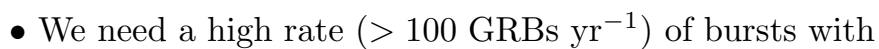
good locations, in order to change the sociology of groundbased optical and radio observations. This many good GRB positions to follow-up each year would make it possible to propose and carry out GRB afterglow monitoring programs at many medium-to-large aperture telescopes.

- The diversity of GRBs, GRB afterglows, and host galaxies means that we need a large number $(>1000)$ of good GRB positions in order to be able to study the correlations between these properties. This is important for determining whether or not there are distinct subclasses of bursts, and more than one burst mechanism. Any correlations found will also impose important constraints on burst mechanisms and models.

- We need many rapid (near real time) one arcminute GRB positions in order to determine whether or not significant optical emission accompanies the bursts (Park 1999), and to make it possible to take spectra of the burst afterglows while the afterglows are still bright - and thereby obtain redshifts of the bursts themselves from absorption line systems, and if there are bursts at high redshifts, from the Ly $\alpha$ break.

- All of the GRBs that BeppoSAX has detected are "long" bursts. Currently we know nothing about the afterglow properties, the distance scale, and the hosts (if any) of "short" bursts. Therefore we need good/quick positions for short bursts, in order to determine these properties for short bursts in the same way that BeppoSAX has enabled us to determine these properties for long bursts.

- Currently, there is a largely unexplored gap in our knowledge of the X-ray and optical behavior of burst afterglows of $\sim 10^{4}-10^{5}$ seconds immediately following the bursts, corresponding to the time needed to bring the BeppoSAX NFIs to bear on a burst. We need to fill in this unexplored gap, in order to see if bursts always, often, or rarely join smoothly onto their X-ray and optical afterglows, and to explore the geometry and kinematics of GRB afterglows (Sari 1999).

- We also need to search for variability in the X-ray and optical afterglows. Observations of such variability would impose severe constraints on models, including the widelydiscussed relativistic fireball model of burst afterglows (see, e.g., Fenimore 1999).

Acknowledgements. The Rome Workshop provided a feast of observational and theoretical results, and the opportunity to discuss them. On behalf of all of the Workshop participants, I would like to thank Enrico Costa, Luigi Piro, Filippo Fontana, and everyone else who helped to organize this meeting for bringing all of us together and for providing us with such "fine dining."

\section{References}

Barthelmy S.D., Butterworth P., Cline T.L., Gehrels N., 1999 (these proceedings)

Belli B.M., 1997, ApJ 413, 281

Belli B.M., 1999 (these proceedings)

Bisnovatyi-Kogan G.S., Timokhin A.N., 1997, Astron. Rep. 41, 483

Bloom J., et al., 1998, ApJ 507, L25

Bloom J., et al., 1999 (these proceedings)

Bulik T., Belczynski K., 1999 (these proceedings)

Cappellaro E., et al., 1997, A\&A 322, 431

Castander F.J., Lamb D.Q., 1998, in Gamma-Ray Bursts, Meegan C.A., Preece R.D., Koshut T.M. (eds.). New York: AIP, p. 520

Castander F.J., Lamb D.Q., 1999, ApJ (in press)

Costa E., et al., 1997, IAU Circ. 6576

Dar A., 1999 (these proceedings)

Dermer C., 1999 (these proceedings)

Djorgovski S.G., et al., 1999, invited talk at the Santa Barbara Workshop on "Gamma-Ray Bursts and their Afterglows"

Fargion D., 1999 (these proceedings)

Fenimore E.E., 1999 (these proceedings)

Fenimore E.E., et al., 1999, ApJ (in press) (astro-ph/9802200)

Frail D.A., et al., 1997, Nat 389, 261

Frail D.A., 1999 (these proceedings)

Fruchter A.S., 1999 (these proceedings)

Fruchter A.S., et al., 1999, ApJ (in press) (astro-ph/9807295)

Galama T.J., 1999 (these proceedings)

Galama T.J., et al., 1997, IAU Circ. 6584

Galama T.J., et al., 1998, Nat (in press) (astro-ph/9806175)

Graziani C., Lamb D.Q., Marion G.H., 1999a, ApJ (submitted) (astro-ph/9810374) 
Graziani C., Lamb D.Q., Marion G.H., 1999b (these proceedings)

Greiner J., 1999 (these proceedings)

Hogg D.W., Fruchter A.S., 1999, ApJ (in press) (astro-ph/9807262)

Kawai N., et al., 1999 (these proceedings)

Kouveliotou C., et al., 1993, ApJ 413, L101

Kouveliotou C., et al., 1996, AIP Conf. Proc. 384, 42

Kulkarni S., 1999 (these proceedings)

Kulkarni S., et al., 1998, Nat 393, 35

Lamb D.Q., Graziani C., Smith I.A., 1993, ApJ 413, L11

Lamb D.Q., Castander F.J., Reichart D.E., 1999 (these proceedings)

Lamb D.Q., Reichart D.E., 1999a, ApJ (submitted)

Lamb D.Q., Reichart D.E., 1999b, ApJ (submitted)

Loredo T.J., Wasserman I., 1998a, ApJ 502, 75

Loredo T.J., Wasserman I., 1998b, ApJ 502, 108

Madau P., 1999 (these proceedings)

Metzger M., et al., 1997a, IAU Circ. 6655

Metzger M., et al., 1997b, Nat 387, 878

Mészáros P., 1999 (these proceedings)

Mukherjee S., et al., 1999, ApJ (in press) (astro-ph/9802085)

van Paradijs J., 1999 (these proceedings)

Park H.S., 1999 (these proceedings)
Pendleton G., et al., 1998, ApJ (in press)

Perlmutter S., 1999 (private communication)

Pian E., et al., 1998a, GCN Report No. 61

Pian E., Frontera F., Antonelli L.A., Piro L., 1998b, GCN Report No. 69

Pian E., et al., 1999 (these proceedings)

Piro L., et al., 1998, GCN Report No. 155

Piro L., et al., 1999 (these proceedings)

Pizzichini G., 1995, in Proc. XXIV ICRC 81

Rees M.J., 1999 (these proceedings)

Reichart D.E., 1998, ApJ 495, L99

Ricker G., et al., 1999 (these proceedings)

Sari R., 1999 (these proceedings)

Schmidt M., 1999 (these proceedings)

Soffitta P., et al., 1998, IAU Circ. 6884

Tavani M., 1998, ApJ 497, L21

Tavani M., 1999 (these proceedings)

Totani T., 1999, ApJ 511, 41

Timokhin A.N., Bisnovatyi-Kogan G.S., 1999 (these proceedings)

Wijers R., Galama T.J., 1999, ApJ (in press)

Woosley S.E., 1999 (these proceedings)

Yoshida A., et al., 1999 (these proceedings) 